\title{
Epidermal Penetration of Gold Nanoparticles and Its Underlying Mechanism Based on Human Reconstructed 3D Episkin Model
}

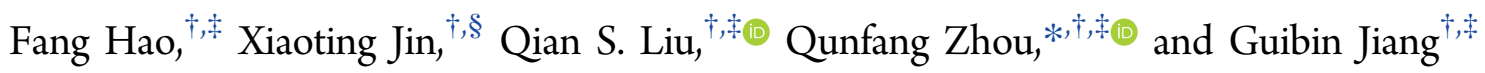 \\ ${ }^{\dagger}$ State Key Laboratory of Environmental Chemistry and Ecotoxicology, Research Center for Eco-Environmental Sciences, Chinese \\ Academy of Sciences, Beijing 100085, China \\ ${ }^{\ddagger}$ College of Resources and Environment, University of Chinese Academy of Sciences, Beijing 100049, China \\ ${ }^{\S}$ Institute of Biomedical Sciences, Shanxi University, Taiyuan 030006, China
}

Supporting Information

ABSTRACT: Nanomaterials are widely used in diverse aspects, and their translocation behavior through the skin would be helpful in understanding the corresponding exposure risks. To reveal how surface functionalization of nanoparticles influences the skin penetration, three kinds of gold nanoparticles (GNPs) with negatively, neutrally, and positively charged surfaces, that is, cetyltrimethylammonium bromide-coated GNPs (CTAB@ GNP), polyvinylpyrrolidone-coated GNPs (PVP@GNP), and citratecoated GNPs (Citrate@GNP), were studied using human reconstructed 3D Episkin model. The measurement of Au distribution in diverse layers of the Episkin model indicated that all three GNPs could penetrate through the epidermis, wherein CTAB@GNP with positive surface charges exhibited the highest efficiency in skin penetration. The upward osmosis of the medium proteins confirmed the occurrence of skin permeation induced by GNP treatment, and the lipid network in the stratum corneum

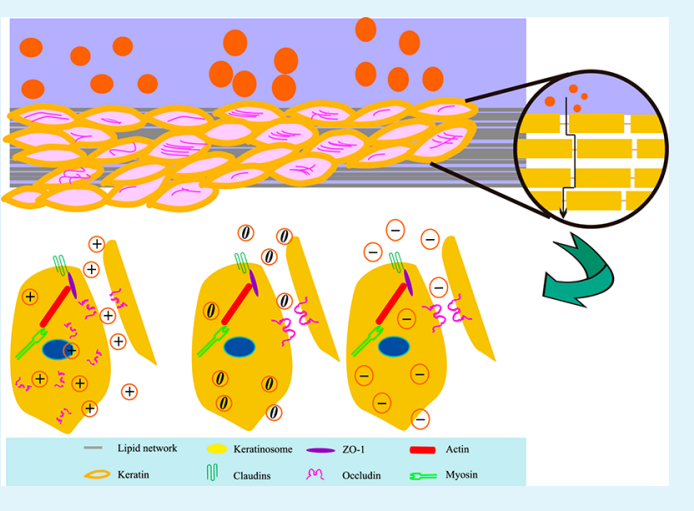
was also altered as the consequence of GNP exposure. When compared to Citrate@GNP and PVP@GNP, CTAB@GNP significantly compromised the tight junction of keratinocytes, causing paracellular penetration of nanoparticles. The existence of cytoplasmic gold showed the transcytosis pathway through endocytosis and exocytosis processes was the main epidermic penetration behavior of the tested GNPs. The study on GNP penetration process through the 3D Episkin model has, on one hand, offered a promising approach to evaluate the translocation process of nanoparticles across the skin, and, on the other hand, provided mechanism explanation for diverse penetration behaviors of GNPs with different surface charges. The findings herein would be of great help in nanotechnology improvement and nanosafety evaluation.

KEYWORDS: gold nanoparticles, 3D Episkin model, epidermal penetration, lipid network, paracellular and transcellular transport

\section{INTRODUCTION}

The application of nanomaterials has been expanding in multiple aspects, such as medical vehicle, imaging probe, and daily supply additives, etc., thus causing potential exposure risk of the environment and human beings. ${ }^{1-4}$ Both in vivo and in vitro experiments have indicated that nanomaterials may cause diverse toxicities, ${ }^{5-10}$ which make nanosafety an important issue, and nanoimpact is now being hotly debated. Understanding how nanomaterials enter into bodies would be of great importance in explaining their potential toxicological effects.

The skin is the largest organ in the human body, ${ }^{11}$ which offers the most extensive exposure interface for the exogenous substances from diverse sources. Among its main constituting layers, that is, epidermis, dermis, and subsidiary organs, ${ }^{12}$ the epidermis is the outer layer of the skin, which provides the substantial barrier function and keeps the epidermal homeostasis. ${ }^{13}$ As shown in Figure S1, the epidermal layer contains four types of sublayers, that is, stratum corneum, stratum granulosum, spinous layer, and basal layer from top to the bottom, and the maintenance of the epidermis depends on differentiation and simultaneous migration of keratinocytes from the bottom to the top surface. ${ }^{11}$ Considering the potential usage of nanomaterials in some specific products like skin care pharmaceuticals, etc., and other unintended exposure routes, the study on their permeation behavior through the epidermis would be helpful in guiding their design and risk assessment.

Previous studies have indicated that different nanomaterials with distinctive physiochemical characteristics could exhibit unique biological behaviors, including skin penetration. ${ }^{11,14,15}$ Some nanoparticles (NPs) hardly penetrate the skin barrier, ${ }^{16-18}$ while others were found to exert transdermal delivery capacities based on both in vitro and in vivo studies, ${ }^{19-21}$ indicating the controversial findings on the penetration behaviors of NPs through the skin barrier. Because

Received: September 9, 2017

Accepted: November 17, 2017

Published: November 17, 2017 
of the high stability of gold nanoparticles (GNPs), they are considered as an excellent fine particle model for skin penetration, and have been extensively studied in this area. For example, Fernandes et al. ${ }^{15}$ compared the penetration behavior of gold nanomaterials with different shapes, and found GNPs were less efficient in epidermal penetration than gold nanorods. The size effect of GNPs on skin penetration was also discussed on the basis of the excised epidermis model, ${ }^{22}$ and small GNPs were found to penetrate more easily into the deep layer of epidermis through paracellular pathway than large ones. The computer simulation studies also showed the important distinction in nanoparticle size-related translocation and the permeation of GNPs through model skin lipid membrane. ${ }^{23}$ Apparently, substantial data have confirmed GNPs could penetrate the epidermis through diverse routes. Surface modification with diverse ligands could regulate different properties of NPs, like surface charges, hydrophobicityhydrophilicity, etc., for multiple purposes, and how surface charges influence their penetration behavior still remains blur. What is more, although ex vivo experiments based on excised epidermis model could help to provide useful information on nanomaterial induced biological processes, their feasibility in high throughput assay still faces lots of challenges, and alternatives to animal studies are now mandatory in dermocosmetic and other related industries. Three-dimensional (3D) cell cultures at tissue or organ levels, which could provide more solid data on explaining the real scenario in vivo than two-dimensional (2D) monolayer cultures, are now being extensively explored and urgently encouraged.

A novel commercial available human reconstructed 3D Episkin model is an in vitro epidermis model with gas-liquid culture (Figure S2), which has been recommended as a uniform alternative by the Organization for Economic Co-operation and Development (OECD) for testing in vitro permeability. ${ }^{24}$ Pharmaceutical and dermocosmetic industries are now using this model as an efficient prediction tool for diverse research and development tests, such as skin-penetration rates of various agents, inflammatory reactions, effects of UV irradiation, and hormone metabolism. ${ }^{25}$ In toxicological studies, this Episkin model is now being increasingly investigated as well. For example, Abdallah et al. ${ }^{26}$ found $\log K_{\text {ow }}$ was the main cofactor for the percutaneous penetration of hexabromocyclododecanes (HBCD) and tetrabromobisphenol A (TBBPA) in the Episkin model, showing its feasibility and relevance as a biologically active barrier for in vitro assays. Therefore, this human reconstructed 3D Episkin model is a very promising approach, and has high potentials in nanotoxicological studies.

In this Article, we used a human reconstructed 3D Episkin model and tested the epidermic permeation for three kinds of GNPs with different surface charges. The downward penetration of GNPs through the epidermis and the upward osmosis of the medium protein were influenced by the surface functionalization of GNPs, wherein cetyltrimethylammonium bromide coated GNPs (CTAB@GNP) with the positively charged surface exhibited the most efficient skin penetration behavior among all. GNP exposure could influence lipid structure, and penetrate the epidermis by both paracellular route and transcellular pathway. The research on surface functionalization-related penetration process of GNPs through the 3D Episkin model has provided a promising approach to assess the translocation of nanoparticles through the skin, and revealed the underlying mechanisms during this biological process.

\section{MATERIALS AND METHODS}

2.1. Reagents. The stock solution of Citrate@GNP ( $5 \mathrm{~nm}, 63 \mu \mathrm{g} /$ $\mathrm{mL}$ ) was purchased from BBI Solutions (UK). The other two coating materials, including polyvinylpyrrolidone (PVP 10, >99\%) and cetyltrimethylammonium bromide ( $\mathrm{CTAB},>99 \%)$, were obtained from Sigma-Aldrich. All other chemicals or reagents used in this study were specified below.

2.2. Surface Modification and Characterization of GNPs. Surface functionalization of GNPs was performed on the basis of the method of ligand exchange. First, $0.5 \mathrm{~mL}$ of $10 \mathrm{mM} \mathrm{CTAB}$ was slowly added to $6 \mathrm{~mL}$ of $21 \mu \mathrm{g} / \mathrm{mL}$ Citrate@GNP aqueous solution under vigorous stirring during $90 \mathrm{~s}$ to obtain CTAB@GNP. As for PVP@ GNP, $10 \mathrm{~mL}$ of $63 \mu \mathrm{g} / \mathrm{mL}$ Citrate@GNP was boiled for 5 min with drop addition of $1 \mathrm{~mL}$ of $40 \mathrm{~g} / \mathrm{L}$ PVP 10 . The synthesized reaction mixtures were purified by ultrafiltration $(5 \mathrm{KD}$ ultrafilter, Millipore, U.S. $)$ at $4{ }^{\circ} \mathrm{C}(3500 \mathrm{rpm}, 20 \mathrm{~min})$ to remove the free ligands. The concentrations of the prepared GNP solutions were determined by inductively coupled plasma mass spectrometry (ICP-MS, Agilent 8800, U.S.). The working solutions were diluted to $10 \mu \mathrm{g} / \mathrm{mL}$ and stored at $4{ }^{\circ} \mathrm{C}$ until use.

The morphologies of three GNPs were observed by transmission electron microscopy (TEM, JEOL, Japan) using 230 mesh copper grid coated with carbon membrane. Their sizes based on TEM characterization were evaluated and fitted with Gauss Distribution. The UV-vis spectra of GNPs were analyzed by scanning the range of 450-650 nm at room temperature using a Shimadzu UV-2450 spectrophotometer (Tokyo, Japan). The surface charges and hydrodynamic sizes were measured by Zeta Sizer Nano ZS (Malvern, UK), and illustrated by zeta potentials and hydrated particle sizes, which were re-evaluated after 7 days storage to confirm the stabilities of the prepared GNPs.

2.3. Configuration and Culture of Human Reconstructed 3D Episkin Model. The kit of 3D Episkin reconstructed human epidermis model was purchased from Shanghai Episkin Biotechnology Co., Ltd. (China), and the experimental setup was shown in Figure S2. It was based on the transwell-like chamber, consisting with the inset and the 12-well plate. The insert contained two layers, that is, the bottom collagen layer as the support material and the upper epidermis layer as the tested target. In each well, $1.5 \mathrm{~mL}$ of the commercially supplied culture medium (Shanghai Episkin Biotechnology Co., Ltd., China) was added to maintain the normal proliferation and differentiation of the keratinocytes. The cell condition during the transportation was ensured by checking the attached temperature tag (less than $40{ }^{\circ} \mathrm{C}$ ), and the cell culture was performed overnight at 37 ${ }^{\circ} \mathrm{C}$ in $5 \% \mathrm{CO}_{2}$ and $100 \%$ humidity. The epidermis cell cultures could be maintained for at least 1 week according to the supplier's instruction. The quality control of the product was strictly guaranteed, and necessary characterization for morphology, half inhibitory concentration $\left(\mathrm{IC}_{50}\right)$, and histological scores were performed and provided along each batch of the kits by the supplier (Table S1).

2.4. GNP Exposure to 3D Episkin Model. Before exposure, the insets containing the collagen and epidermis were switched to the commercially available test medium (Shanghai Episkin Biotechnology Co., Ltd., China), and maintained for $2 \mathrm{~h}$. The top interface of epidermis was evenly exposed to $10 \mu \mathrm{L}$ of $10 \mu \mathrm{g} / \mathrm{mL}$ GNP solutions for $2 \mathrm{~h}$, and the negative control was set by adding $10 \mu \mathrm{L}$ of Milli- $\mathrm{Q}$ water. After exposure, the top interface of epidermis was washed by $100 \mu \mathrm{L}$ of Dulbecco's phosphate buffered saline (DPBS, Gibco), and the surface solution was transferred and stored at $-80{ }^{\circ} \mathrm{C}$ for subsequent protein analysis. Five-time wash with DPBS was performed to completely remove excessive GNPs on the epidermis surface. The bottom medium $(0.5 \mathrm{~mL})$ was also collected for daily monitoring of Au contents. The Episkin culture was continued by transferring the insets back to the culture medium for $22 \mathrm{~h}$. The exposure, sample collection, and subsequent culture were carried out in a similar way for a second and third time. After the third time $22 \mathrm{~h}$ culture, the exposure experiments were terminated, and the tissue samples were punched off from inserts, and submitted to direct analysis using attenuated total reflection Flourier transformation infrared spectroscopy (ATR-FTIR) and differential scanning calorimetry (DSC), or snap frozen in liquid 
nitrogen and subsequently stored at $-80{ }^{\circ} \mathrm{C}$ for ICP-MS analysis, SDS-PAGE separation, RT-PCR, and Western blot assays, or fixed in $10 \%$ buffered formalin (Sinopharm, China) for imaging mass spectrometry analysis, histopathological observation, and immunofluorescence assay, or fixed in $2.5 \%$ glutaraldehyde $(\mathrm{pH} \mathrm{7.3,}$ Sinopharm, China) containing $0.1 \mathrm{M}$ sodium cacodylate (Sinopharm, China) for scanning transmission electron microscopy coupled with energy dispersive system (STEM-EDS) analysis. As for medium samples, $0.5 \mathrm{~mL}$ of the test medium was kept at $-20{ }^{\circ} \mathrm{C}$ until $\mathrm{Au}$ analysis.

2.5. ICP-MS Analysis for GNP Distribution. The tissue samples, including the epidermis and the collagen layers collected from the inset, were shortly warmed at $56^{\circ} \mathrm{C}$, and the collagen matrix was torn off from the epidermis layer. After weighing, these two kinds of samples were dissolved in $1 \mathrm{~mL}$ of aqua regia, digested at room temperature for $12 \mathrm{~h}$, and then heated at $95{ }^{\circ} \mathrm{C}$ until the solution volumes were less than $0.5 \mathrm{~mL}$. The residue was diluted with Milli-Q water to the final volume of $20 \mathrm{~mL}$. The as-prepared samples were submitted to the analysis of Au contents using Agilent 8800 ICP-MS (U.S.), and the results were expressed as $\mathrm{ng} / \mathrm{g}$ in epidermis and collagen matrix samples. As for the analysis of Au contents in the test medium, $0.5 \mathrm{~mL}$ of samples was processed and analyzed in a way similar to that described above.

The decrease ratio in the test medium was calculated using the following equation:

$$
C_{\text {day } 1}-\left(C_{\text {day2or3 }}\right) / C_{\text {dayl }}
$$

where $C_{\text {day1 }}$ means $\mathrm{Au}$ concentration in the test medium collected on exposure day 1 , and $C_{\text {day2or3 }}$ means Au concentration in the test medium collected on exposure day 2 or 3 .

2.6. Imaging Mass Spectrometry Analysis for GNP Penetration in Epidermis. The epidermis samples from different exposure groups were fixed in $10 \%$ buffered formalin for at least $24 \mathrm{~h}$, and dehydrated with a series of sucrose solutions $(10 \%, 20 \%$, and $30 \%)$ for $12 \mathrm{~h}$ in each step. The samples were then immersed in OCT embedding medium (SAKURA, U.S.), and placed in liquid nitrogen for $1 \mathrm{~min}$. The as-prepared tissue samples were frozen sectioned to 40 $\mu \mathrm{m}$ at $-20{ }^{\circ} \mathrm{C}$ using a Leica HM 525 cryostat microtome (Germany), and submitted to laser ablation ICP-MS (LA-ICP-MS) analysis. GNP penetration through epidermis was evaluated using a NWR 213 laser ablation system (NEW WAVE, U.S.) coupled with a NexION 300D ICP-MS instrument (Perkin Elemer, U.S.). Ablation carrier gas was helium, and the flow rate was $0.8 \mathrm{~L} / \mathrm{min}$. Argon was introduced through a Y-piece at the typical flow rate of $0.6 \mathrm{~L} / \mathrm{min}$. The optical operating conditions were given in Table S2.

2.7. Protein Analysis Based on SDS-PAGE and LC-MS/MS. The solution samples daily collected on the top of epidermis after GNP exposure were quantitatively analyzed for protein concentrations using BCA assay (Thermo Fisher, U.S.). After concentration normalization, the protein profiles from different exposure groups were characterized by SDS-PAGE separation (Biorad, U.S.) coupled with silver staining (Beyotime Biotechnology, China). The main protein bands in SDS-PAGE were cut off, and the protein structures were unfolded with dithiothreitol (DTT) and iodacetamide (IAM) solution (Sigma, U.S.). After dehydration with acetonitrile, the gel samples were overnight digested by trypsin. The digested samples were submitted to LC-MS/MS analysis (Bruker Daltonics, U.S.) for protein identification. The software used for protein search was MASCOT (version 2.3.01), and the parameters were listed in Table S3.

The exosmosis protein amounts in surface solutions were compared in different exposure groups after the first time GNP exposure based on protein concentrations measured by BCA kit (Thermo Fisher, U.S.).

2.8. Histopathological Analysis. The epidermis samples from different exposure groups were fixed and dehydrated in a way similar to the sample pretreatment for LA-ICP-MS analysis. The tissues were embedded in paraffin, sectioned to $4 \mu \mathrm{m}$, and submitted to hematoxylin and eosin staining ( $\mathrm{H} \& \mathrm{E}$, Sinopharm, China). The as- prepared sections were observed and pictured under a microscope (Olympus IX73, Japan) at 20× magnification.

2.9. Lipid Network Analysis Using ATR-FTIR. The fresh epidermis samples were carefully placed on the slides and wiped by filter paper, which were dried overnight in a desiccator. The complete stretched samples were then placed on an ATR diamond crystal for the subsequent measurements. The spectra were scanned using a Thermo spectrophotometer (Nicolet 5700, U.S.) with the wavenumber ranging from 500 to $3400 \mathrm{~cm}^{-1}$, the scan resolution was $4 \mathrm{~cm}^{-1}$, and a total 128 scans were controlled. The raw data were processed using Savitsky-Golay function.

2.10. Crystal Transition Analysis Using DSC. The specimens from ATR-FTIR experiments were folded into pellets, which were subsequently placed into aluminum pans and crimped, and then added in a test calorimeter. DSC heat flow curves were measured on a DSC 8000 system (Perkin Elemer, U.S.) from 0 to $120^{\circ} \mathrm{C}$, and the reverse progress was controlled from 120 to $0{ }^{\circ} \mathrm{C}$. The heating or cooling rate was $10^{\circ} \mathrm{C} / \mathrm{min}$. The baseline was scanned using two empty pans to eliminate any thermo interferences. The data were finally analyzed by Pyris software.

2.11. RT-PCR for Transcriptional Levels of Tight Junction and Exocytosis-Related Proteins. Total RNA was extracted from epidermis samples by Trizol reagent (Invitrogen, U.S.) and purified following the commercially recommended protocol. The reverse transcription of mRNA to cDNA was performed by one-step iScript cDNA Synthesis Kit (Biorad, U.S.). The cDNA samples were diluted by 10 -fold, submitted to RT-PCR analysis using SYBR Green Supermix kit on a Roche 480 system (Roche, UK). The tested genes included occludin, zonula occludens protein 1 (ZO-1), claudin, ATP-binding cassette transporter subfamily member C1 (ABCC1), vesicle secretion-related TRAPP, divalent metal transporter 1 (DMT1), copper transporting ATPase (ATP7A, ATP7B), and glyceraldehyde-phosphate dehydrogenase $(\mathrm{GAPDH})$. The related forward and reverse primers were listed in Table S4. The relative transcriptional levels of the target genes were normalized to GAPDH mRNA expression and calculated by the $2^{-\Delta \Delta \mathrm{CT}}$ method.

2.12. Western Blot Analysis. The epidermis samples were homogenized in liquid nitrogen and lysed with $200 \mu \mathrm{L}$ of lysis buffer (10 mM Tris- $\mathrm{HCl}$ buffer, containing $2 \mathrm{mM} \mathrm{NaF}, 1 \%$ cocktail, $1 \mathrm{mM}$ $\mathrm{Na}_{3} \mathrm{VO}_{4}$, and $\left.0.6 \mathrm{mM} \mathrm{H}_{2} \mathrm{O}_{2}, \mathrm{pH} 7.4\right)$. After $3 \mathrm{~h}$ extraction, the samples were centrifuged $(12000 \mathrm{~g})$ at $0{ }^{\circ} \mathrm{C}$ for $15 \mathrm{~min}$. The supernatants were quantitatively analyzed for protein normalization, and processed for SDS-PAGE analysis. Equal amount of protein sample from each exposure group was loaded and separated on the denaturing gradient gel (BioRad, U.S.) for $1 \mathrm{~h}$, and transferred to a $0.22 \mu \mathrm{m}$ nitrocellulose membrane (Millipore, U.S.). The membrane was blocked for $2 \mathrm{~h}$, and separately incubated with different primary antibodies overnight at 4 ${ }^{\circ} \mathrm{C}$. The primary antibodies used in this study were antioccludin (1:1000, Abcam, U.S.), anticlathrin (1:1000, Abcam, U.S.), anticaveolin-1 (1:1000, Abcam, U.S.), and anti-GAPDH antibodies (1:1000, Abcam, U.S.). The subsequent processes included the incubation with the corresponding second antibodies (peroxidaseconjugated goat antirabbit second antibodies from ZSGB-BIO, 1:2500; China) and the development for the target protein expressions (Pierce ECL, Thermo Scientific, U.S.). The pixel densities on the developed $\mathrm{X}$-ray film were evaluated by Quantity One software for the target protein expression levels.

2.13. Immunofluorescence Assay for Occludin. The fixed epidermis samples were processed in a way similar to the sample pretreatment for LA-ICP-MS analysis. The frozen sections $(5 \mu \mathrm{m})$ were washed with PBS for 5 min under slight rotatory shaking. After a brief air-dry, the sections were immersed into $3 \%$ hydrogen peroxide for $15 \mathrm{~min}$, and washed with PBS three times (5 min each). The antigen repair was performed by incubating the section samples with trypsin at $37{ }^{\circ} \mathrm{C}$ for $5 \mathrm{~min}$. After PBS wash, $10 \%$ goat serum (VWR Shanghai Co., Ltd., China) was applied to block the nonspecific binding sites. The samples were subsequently incubated with the primary antioccludin antibody (1:50, Abcam, U.S.) at $4{ }^{\circ} \mathrm{C}$ overnight, followed by $1 \mathrm{~h}$ incubation of Alexa 488 labeled antirabbit second antibody (1:100, ZSGB-BIO, China). The slides loaded with section 
(A)
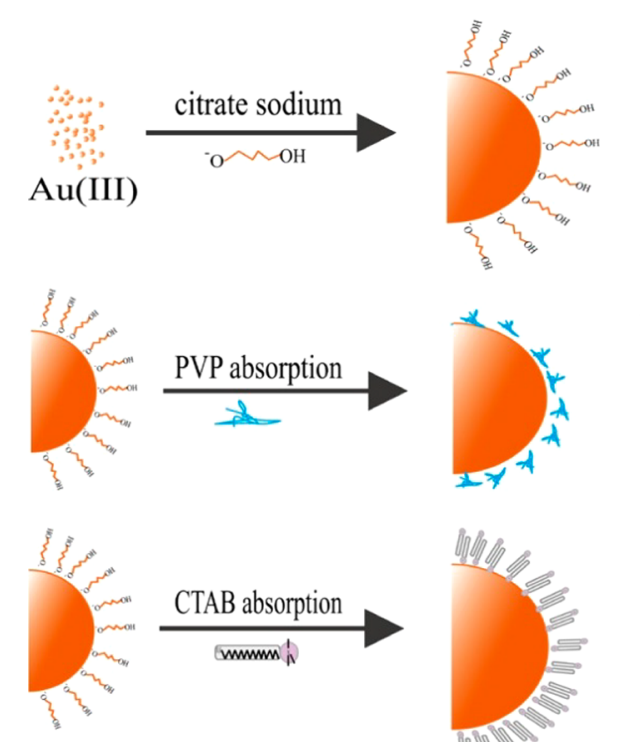

(B)
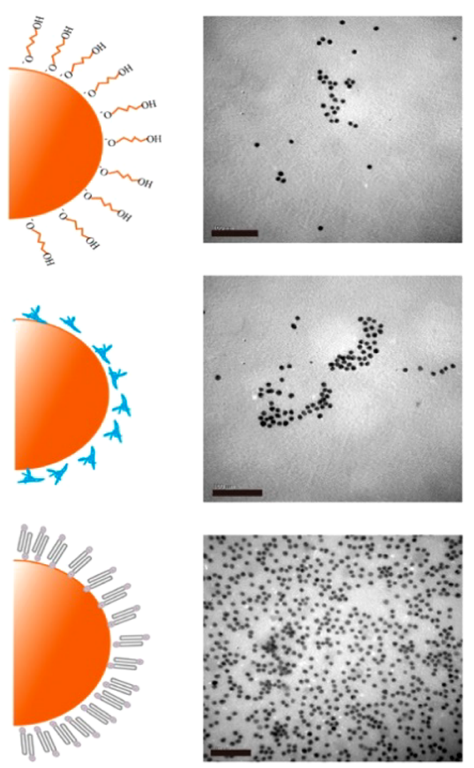

(C)
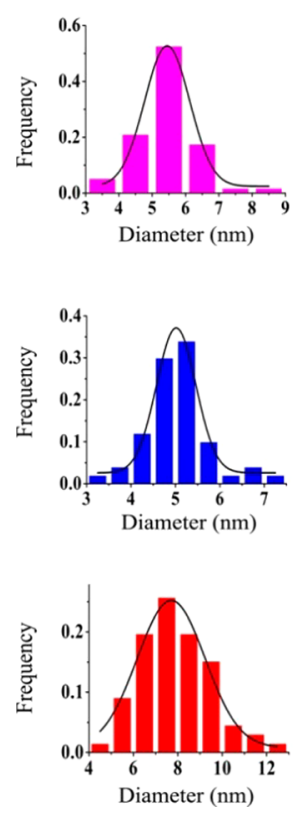

(D)
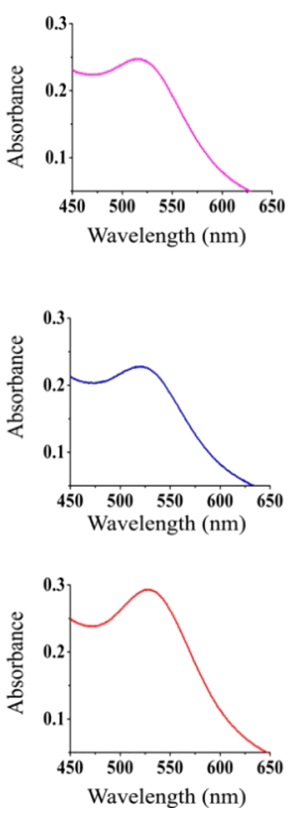

Figure 1. Characterization of GNPs. (A) The cartoon sketch for surface functionalization of GNPs. TEM images (B) and the statistic particle size distributions (C) of the GNPs. (D) UV-vis absorption spectra of GNPs. Scale bars represent $100 \mathrm{~nm}$.
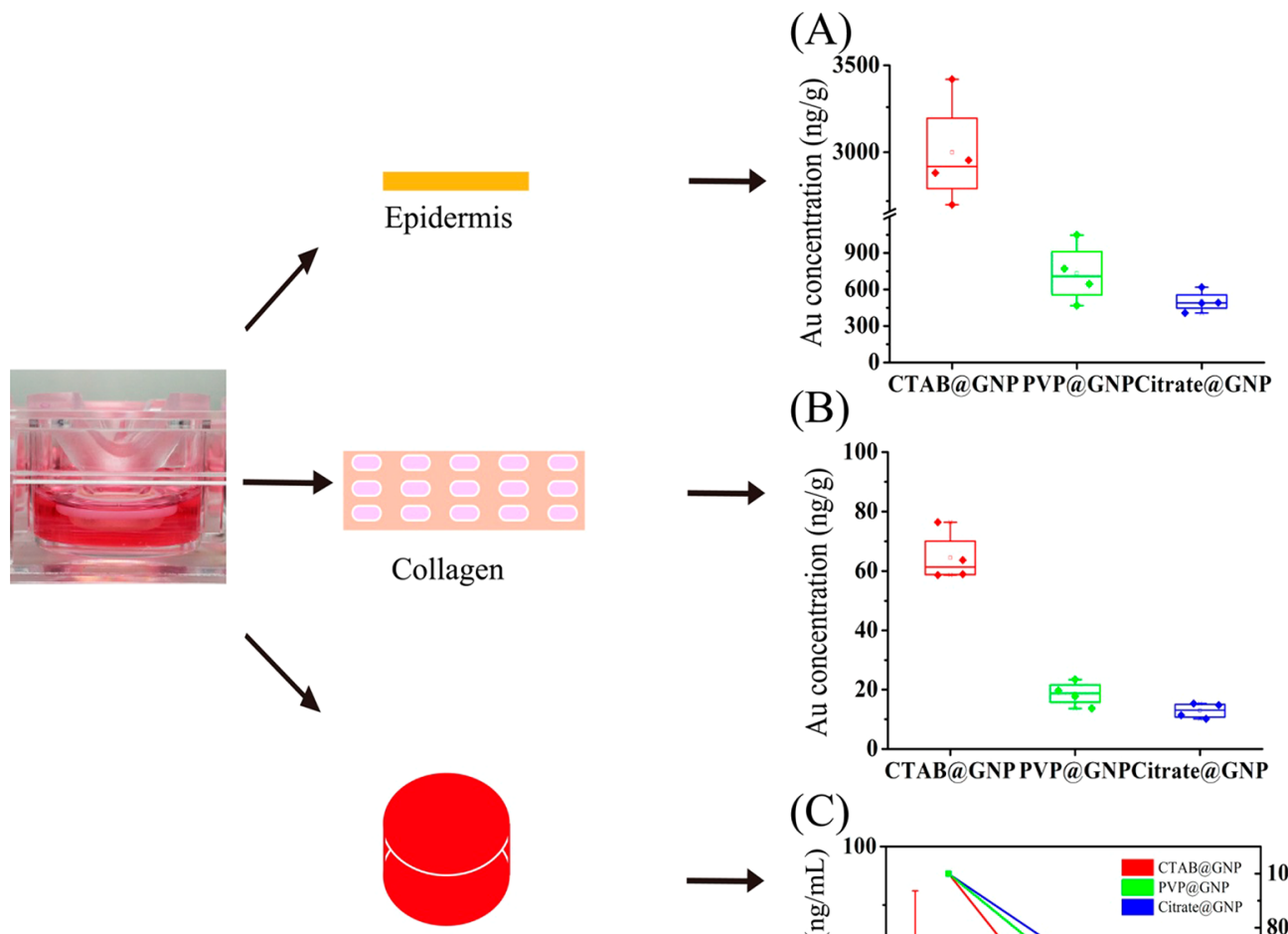

(C)

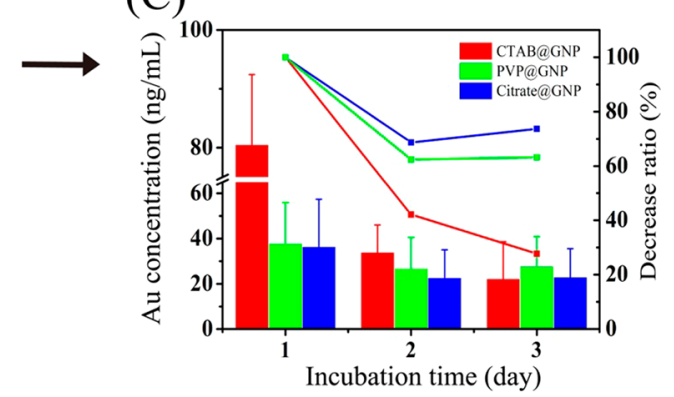

Figure 2. GNP distribution in the Episkin model. Au concentrations in epidermis (A) and collagen layers (B) collected on exposure day 3. (C) Au concentrations in the test medium during 3-day exposure. The decrease trend lines were calculated relative to Au levels in three GNP treatment groups on exposure day 1. 
samples were sealed with antifade mounting medium containing DAPI (VECTOR, U.S.), and stored in the dark at $4{ }^{\circ} \mathrm{C}$. The samples were observed and photographed using a Leica TCS-SP5 confocal laser scanning microscope (Germany).

2.14. Elemental Au Distribution Based on STEM-EDS Analysis. The epidermis samples from different exposure groups were cut into $1-2 \mathrm{~mm}^{3}$ pieces and immediately immersed into $2.5 \%$ glutaraldehyde containing $0.1 \mathrm{M}$ sodium cacodylate ( $\mathrm{pH}$ 7.3). After 24 $\mathrm{h}$, the specimens were post fixed in osmium tetroxide buffer (Sigma, U.S.) for $1 \mathrm{~h}$. After being washed, the tissue samples were immersed into uranyl acetate for $20 \mathrm{~min}$, and subsequently dehydrated in a series of ethanol solutions. After embedding into epoxy resin, $100 \mathrm{~nm}$ ultrathin sections were prepared using a slicing machine (Leica UC7, Germany). The ultrastructure of the specimen and elemental $\mathrm{Au}$ distribution were photographed and analyzed under a F20 fieldemission STEM coupled with EDS (FEI, U.S.).

2.15. Statistical Analysis. Each experiment condition was performed in triplicate, and all assays were independently performed three times or more. The data were represented as mean \pm standard deviation. The significant difference between the negative control and the exposure groups was denoted by a $p$ value less than $0.05\left(^{*}\right)$ or $0.01(* *)$ using $t$-test.

\section{RESULTS AND DISCUSSION}

3.1. Characterization of GNPs. Citrate@GNP was used to prepare PVP- and CTAB-coated GNPs (i.e., PVP@GNP, CTAB@GNP) by surface charge modification as revealed by the synthesis strategy in Figure 1A. TEM observation indicated that the initial Citrate@GNP was spherical, and the particle shape did not obviously change during the subsequent ligand exchanging reactions (Figure 1B). The results from TEM images fitted with Gauss function showed that the particle sizes of both Citrate@GNP and PVP@GNP were around 5 nm, while that of CTAB@GNP was around $7.68 \mathrm{~nm}$ (Figure 1C). Considering CTAB molecular was $2 \mathrm{~nm}$ in length and its bilayer was about $3 \mathrm{~nm}$, this result was consistent with the theoretical calculation. Similar findings on surface ligands influencing particle sizes were previously reported, ${ }^{27-30}$ and $\mathrm{CTAB}$ was found to have a relatively shorter carbon chain and lower molecule weight, when compared to the other positive charge regulating ligands, like polyethylenimine (PEI), polyethylene glycol (PEG), and poly(diallyldimethylammonium chloride) (PDADMAC). It was thus selected herein to regulate the positive surface charge of GNPs, avoiding too much change in particle sizes. The zeta potentials of Citrate@GNP, PVP@ GNP, and CTAB@GNP were -25.70, -5.09, and $26.50 \mathrm{mV}$, respectively (Table S5), representing they had negatively, slightly negatively (regarding as neutral herein), and positively charged surfaces. The dynamic light scattering (DLS) measurement showed that the hydrated sizes were 5.10, 5.85, and 8.76 nm for Citrate@GNP, PVP@GNP, and CTAB@GNP, separately (Table S5), which were well consistent with TEM results (Figure $1 \mathrm{C}$ ). Both zeta potentials and hydrated sizes of these three GNPs were remeasured after 7-day storage to evaluate their stabilities, and the results remained unchanged (Table S6). The surface charges of nanoparticles could guarantee the high stabilities of their aquatic dispersions, ${ }^{11}$ and the as-prepared GNPs in this study were quite stable. The analysis of surface plasmon resonance (SPR) showed all GNPs had the maximum absorption peak at $520 \mathrm{~nm}$ (Figure 1D), showing that surface functionalization did not alter the particle characteristics and their dispersities. ${ }^{22}$ Three kinds of ligands used herein, that is, citrate, PVP, and CTAB, were all hydrophilic, which also endowed GNPs with high aquatic dispersity and good stability in water suspensions.
3.2. Surface Functionalization Influences GNP Penetration. The penetration of GNPs through the Episkin model was evaluated by the analysis of elemental gold distribution in diverse layers, including the epidermis, collagen matrix, and culture medium (Figure 2), using ICP-MS (Agilent 8800). The results showed $\mathrm{Au}$ concentrations in epidermis ranged from 600 to $3000 \mathrm{ng} / \mathrm{g}$ (Figure 2A), and those in collagen were in the range of $15-65 \mathrm{ng} / \mathrm{g}$ (Figure $2 \mathrm{~B}$ ). This was reasonable as the epidermis was the upper layer of the Episkin model closely adjacent to the surface where GNPs were daily administered. When three GNPs were compared, CTAB@GNP with the positive surface charges had the most efficient penetration through the Episkin model, as demonstrated by the relatively high Au concentrations in both epidermis and collagen layers. As for PVP@GNP and Citrate@GNP, comparable penetration was observed with the former one exhibiting a little bit more efficiency. Consistent with the previous finding, ${ }^{15}$ this could be explained by the fact that the surface charge of skin was physiologically negative, and would easily adsorb the particles with positively charged surface. Considering the potential contribution of cytotoxic $\mathrm{CTAB}$ to the skin penetration of GNPs, CTAB@GNP was purified by ultrafiltration to remove free $\mathrm{CTAB}$ molecules, and the observed effect was believed to be attributed to CTAB@GNP itself. A similar particulatespecific hemolytic effect was observed for CTAB coated gold nanorods by comparing to that of the equivalent amount of CTAB small molecular. ${ }^{31}$ On the basis of the calculation of the maximum number of the ligand molecular adsorbed on spherical particle, ${ }^{32}$ the total CTAB coated on GNP was about $0.6 \mu \mathrm{M}$ when the exposure concentration of CTAB@ GNP was $10 \mu \mathrm{g} / \mathrm{mL}$, which was far less than the cytotoxic level of CTAB $(100 \mu \mathrm{M})$ to human skin keratinocytes and fibroblast cells, $^{28,29}$ further revealing the skin penetration of CTAB@ GNP was free from the potential cytotoxicity of the surface ligand. In view of the surface charge-relevant effect, similar findings on the skin penetration of positively charged GNPs were observed when the surface ligands were polyethylene glycol (PEG) ${ }^{15}$ and poly dimethyl diallyl ammonium chloride (PDADMAC), ${ }^{30}$ which had relatively high biocompatibilities, showing positive surface charges did matter.

To reveal the diffusion behavior of GNPs through the epidermis, the cross section from each exposure groups was submitted to LA-ICP-MS (PerkinElmer, U.S.) analysis, and the results were indicated in Figure S3. CTAB@GNP showed apparent permeation through epidermis, which exhibited in a passive diffusion mode from the top to the bottom due to the concentration gradient caused by three-time treatments performed daily (Figure S3A). As for Citrate@GNP with negative surface charges, their penetration seemed rather random and unevenly, with several scattered spots containing relatively high $\mathrm{Au}$ levels (Figure S3B). Considering the repelling force between negatively charged particles and epidermic cells, Citrate@GNP was not readily absorbed, and the limited partial penetration through epidermis could be possibly contributed by the compromised defense function of the skin model. The imaging mass spectrometry for PVP@ GNP exposed samples showed low levels of Au uniformly distributed in epidermis, indicating that limited but even permeation occurred for this kind of nanoparticle (Figure S3C).

To test if GNPs could penetrate through the epidermis and collagen layer, $\mathrm{Au}$ concentrations in bottom medium were daily monitored, and the results in Figure $2 \mathrm{C}$ show that the highest 

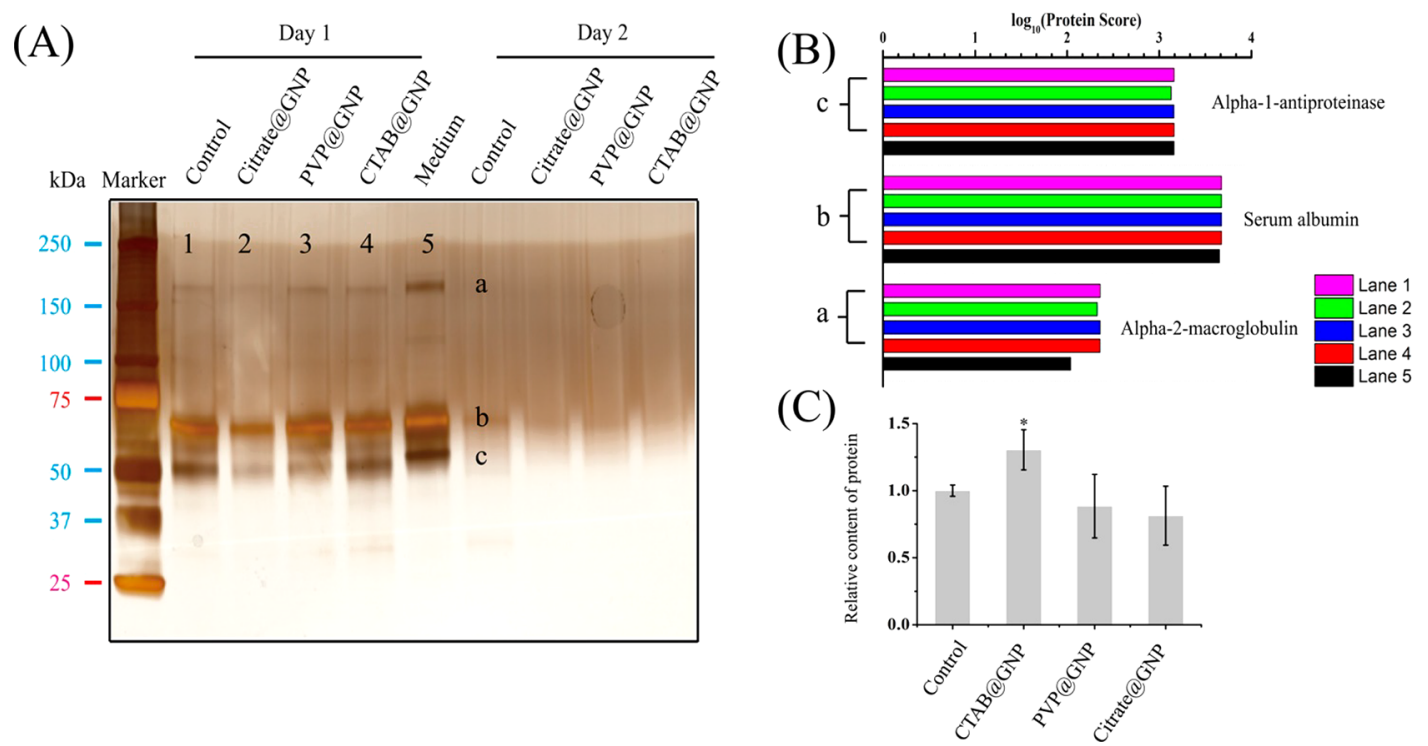

Figure 3. Upward osmosis of medium proteins through the Episkin model. (A) SDS-PAGE analysis of surface solution samples collected on exposure days 1 and 2. (B) Protein identification of bands a, b, and $c$ using LC-MS/MS. (C) The relative protein concentrations in surface solutions on exposure day $1 .{ }^{*} p<0.05$.

Au level (80 ng/mL) was observed in CTAB@GNP exposure group on day 1 , confirming its most efficient penetration through the Episkin model when compared to the other two GNPs. This finding was consistent with what was observed by LA-ICP-MS (Figure S3). As the effect of exposure time was explored, an obvious decreasing trend was observed in medium $\mathrm{Au}$ concentration of CTAB@GNP exposure group, while a slight decrease occurred in those of the other two groups. This could be ascribed to the fact that the keratinocytes of epidermis gradually aged, which could be well characterized by the rapid growth of the epidermis, and the stacked differentiated keratinocytes formed the functionally improved skin barrier as the time prolonged. The upper layer of stratum corneum became thicker as revealed previously, ${ }^{33}$ and the blockage of GNP penetration thus increased.

3.3. GNP Exposure Causes Upward Osmosis through the Episkin. As demonstrated above, GNPs could penetrate through the Episkin model, showing the permeability of the epidermic barrier. Therefore, whether the medium or epidermic contents could effuse to the top surface under concentration gradient force was worthy of investigation. The exposure solutions were collected $2 \mathrm{~h}$ after GNP exposure, and submitted to protein analysis. As indicated by SDS-PAGE analysis in Figure 3A, apparent protein bands with similar profile appeared in all samples on day 1 , while protein levels were nearly undetectable on day 2. This result was consistent with the finding that decreased $\mathrm{Au}$ levels were observed in bottom mediums (Figure 2C) due to the increasing barrier function of the Episkin model. When the protein species were analyzed, the protein profiles in exposure solutions (lane 1-4) were consistent with those in medium (lane 5), wherein three main proteins at the molecular weights of $\sim 160$ (a), $\sim 65$ (b), and $\sim 50 \mathrm{kDa}(\mathrm{c})$ were detected. Protein identification based on liquid chromatography-mass spectrometry/mass spectrometry (LC-MS/MS) coupled with Mascot software analysis showed that they were alpha-2-macroglobulin (a), serum albumin (b), and alpha-1-antiproteinase (c), respectively (Figure 3B), and the protein species in different lanes were exactly the same. Apparently, protein exosmosis from bottom medium to top surface occurred in Episkin models on day 1 due to the imperfect epidermic barrier function.

When the exosmosis protein in exposure solutions collected on day 1 was quantitatively measured, it was found that the concentrations varied with different GNP treatments (Figure 3C). Apparently, the relative protein concentration in CTAB@ GNP exposure group was significantly higher than that in the control $(p<0.05)$, while PVP@GNP and Citrate@GNP exposure groups showed no difference from the control. This result demonstrated that GNPs with positively charged surfaces induced relatively higher permeability of the Episkin model than the others, which was consistent with the results of epidermic and medium $\mathrm{Au}$ concentrations on day 1 (Figure $2 \mathrm{C}$ ). Considering there was no cytoplasmic protein observed in exposure solutions as revealed by Figure $3 \mathrm{~A}$ and $\mathrm{B}$, the exosmosis medium protein detected on exposure day 1 could penetrate the epidermis through the paracellular routes.

3.4. GNP Exposure Induces Structural Changes in Lipid Network of Epidermis. As shown in Figure S1, the layer of stratum corneum lies on the top surface of the epidermis, which is mainly composed of lipid and enucleated keratinocytes from the complete differentiation of keratinocytes. The lipid network is essential in inhibiting the deep diffusion of exogenous chemicals or particles, and controlling material exchange out of the skin. ${ }^{34}$ As GNPs encountered the epidermis, they first contacted the stratum corneum, and the investigation on how NPs passed through the stratum corneum would help to reveal the penetration behavior of GNPs in the epidermis. Histopathological observation revealed that no obvious structural changes occurred in the epidermis, including the stratum corneum layer, upon GNP exposure (Figure S4), suggesting the penetration process of GNPs was independent of histopathological injury or cytotoxicities from surface ligands, as both citrate and PVP had relatively high biocompatibilities, ${ }^{35,36}$ and the CTAB amount coated on GNP was far less than its cytotoxic levels to either keratinocytes ${ }^{29}$ or fibroblasts. ${ }^{28}$ Nevertheless, cell normal function could be compromised by GNP exposure, even if no obvious structural changes were apparently observable. As indicated by Schneider 
(A)

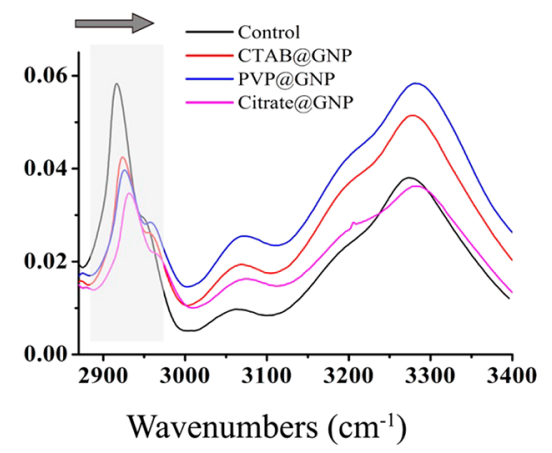

(B)

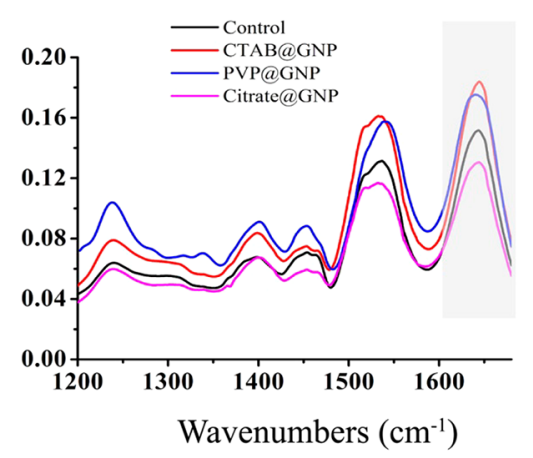

(C)

\begin{tabular}{|c|c|c|c|c|}
\hline \multirow[t]{2}{*}{ Human skin transition } & \multirow{2}{*}{$\begin{array}{l}\text { Transition temperatures } \\
\text { of untreated skin, }{ }^{\circ} \mathrm{C}\end{array}$} & \multicolumn{3}{|c|}{$\begin{array}{c}\text { Transition temperatures of GNP-treated } \\
\text { skin, }{ }^{\circ} \mathrm{C}\end{array}$} \\
\hline & & CTAB & PVP & Citrate \\
\hline I & $46-49$ & -- & $45-47$ & -- \\
\hline II & $65-73$ & $65-73$ & $65-73$ & $65-73$ \\
\hline III & -- & -- & $74-75$ & -- \\
\hline
\end{tabular}

Figure 4. GNP exposure induced lipid network changes in the Episkin model. The deconvolution spectra of the epidermis from 2850 to $3400 \mathrm{~cm}^{-1}$ (A), and from 1200 to $1700 \mathrm{~cm}^{-1}$ (B). (C) The crystal transition analysis of the stratum corneum before and after GNP treatments.

et al., ${ }^{37}$ there were two ways for nanoparticle penetration: (1) the paracellular lipid-stack space of corneocytes and (2) transport into and out of the keratin-rich corneocytes. To study the effects of GNPs on the stratum corneum, its lipid network was characterized by ATR-FTIR and DSC.

As the penetration depth of the evanescent wave from ATRIR was typically between 0.5 and $2 \mu \mathrm{m}$, it could help to capture the spatial organization information on the lipid functional groups in the stratum corneum layer. ${ }^{38}$ As indicated in Figure 4A, the band of $2920 \mathrm{~cm}^{-1}$ in the control, which designated the lipid network in stratum corneum, ${ }^{39}$ was shifted to 2925,2927 , and $2937 \mathrm{~cm}^{-1}$ upon the treatments of CTAB@GNP, PVP@ GNP, and Citrate@GNP, respectively. Considering this band was attributed to the stretching vibrations of ceramides, fatty acid, and cholesterol in stratum corneum, ${ }^{40}$ the band shifts indicated GNPs caused the alterations of lipid lamellae from trans to gauche formation. This transformation was associated with the lipid fluidization of stratum corneum, ${ }^{41}$ and the interaction between GNPs and acyl side chains was the main cause for the transformation in lipid network. Comparatively, Citrate@GNP with negatively charged surface exerted more influences on wavenumber shift than did the other two GNPs, showing their potentially higher interactions with lipid network. Similarly, Wang et al. ${ }^{42}$ reported that the negatively charged nanoparticles could induce the transformation of lipid bilayer interface from semifluidization bilayer to gelled membrane. In contrast, the lipid network could be turned to fluid bilayer by positively charged nanoparticles, ${ }^{43}$ resulting in easier penetration of the positively charged nanoparticles through the bilayer as compared to negatively charged nanoparticles as observed in Figure 2.

The stretching vibration of amide indicated by $1640 \mathrm{~cm}^{-1}$ is mainly from the keratin component in stratum corneum layer. ${ }^{39}$ As shown in Figure 4B, PVP@GNP induced the left shift of this band, representing their interaction with keratin due to the cluster formation between the biocompatible coating of PVP and keratin. Nevertheless, CTAB@GNP and Citrate@GNP did not show any effects.

DSC is a thermoanalytical technique commonly used in the detection of phase transitions, ${ }^{44}$ and typical thermal transitions were previously characterized for stratum corneum membranes from diverse species. ${ }^{45}$ On the basis of monitoring the heat flowing to the epidermis to increase its temperature at the same rate as the reference, the thermal transitions were investigated for both the control and the GNP exposure groups, and the results were depicted in Figure 4C. The DSC thermogram of the untreated control epidermis showed two endothermic transitions at $46-49{ }^{\circ} \mathrm{C}$ (I) and $65-73{ }^{\circ} \mathrm{C}$ (II). Endothermic transition I was associated with the transformation of lipid crystal from orthorhombic form to hexagonal system, and II was attributed to the phase change of hexagonal system to liquid state. ${ }^{46}$ As for CTAB@GNP and Citrate@GNP exposed skin, only endothermic transition II was observed, indicating the original lipid network of epidermis became disorderly. ${ }^{47}$ It was surprising that a new endothermic transition at $74-75{ }^{\circ} \mathrm{C}$ was formed in PVP@GNP exposed samples, which suggested the potential cluster formation due to the interaction of PVP coating with keratin. This result was consistent with the wavenumber shift at $1640 \mathrm{~cm}^{-1}$ in ATR-FTIR analysis (Figure 4B). Altogether, the tested three GNP species apparently exhibited unique characteristics in their interactions with the lipid network and keratin in the stratum corneum layer of epidermis, regarding their distinctive influences characterized by ATR-FTIR and DSC.

3.5. GNP Penetration through Paracellular Route. The stratum granulosum layer lies beneath the stratum corneum in the 3D Episkin model (Figure S1), which provides the substantial barrier function through the cell junctions, ${ }^{48}$ wherein the tight junctions seal up cellular gaps, preventing exogenous pathogen or chemicals from deep invasion. ${ }^{49}$ The 
(A)

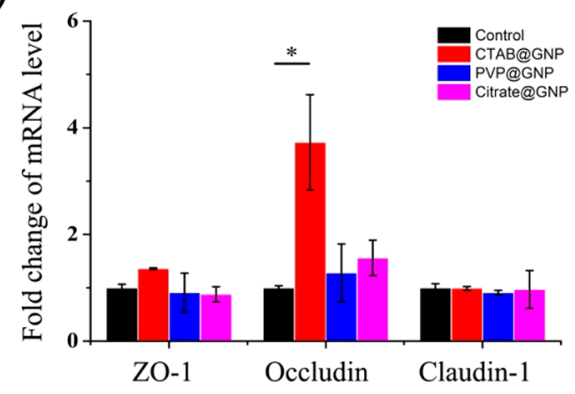

(B)

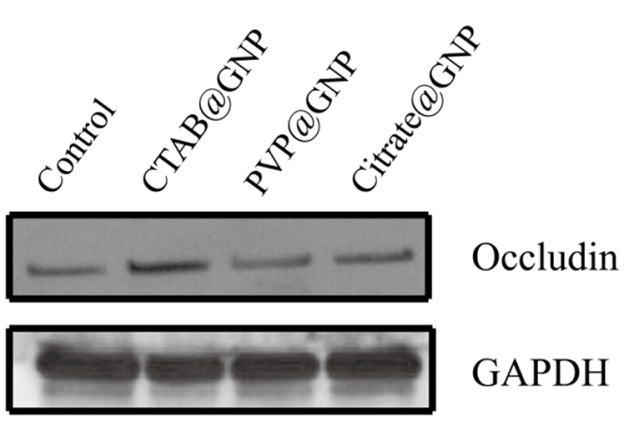

(C)

Control
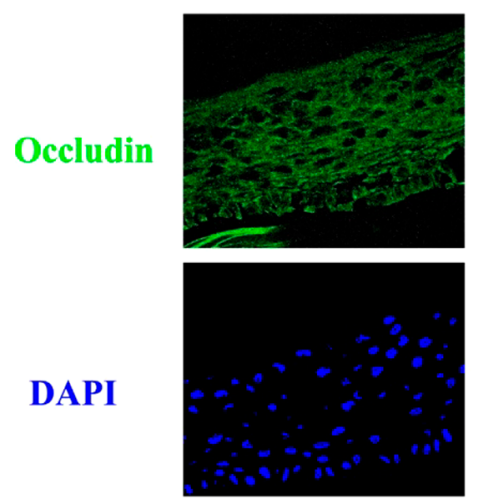

DAPI

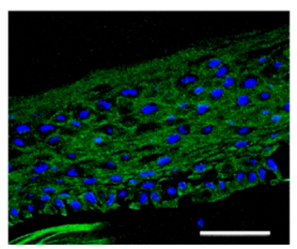

CTAB@GNP
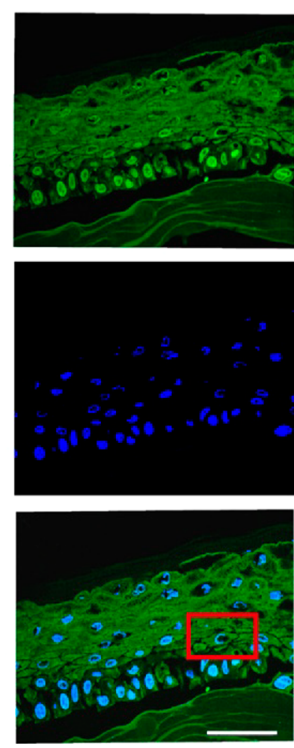

PVP@GNP
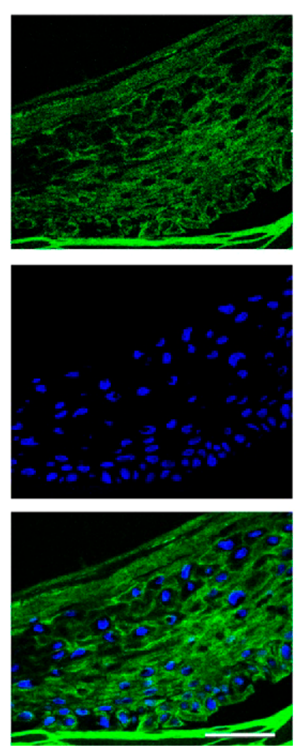

\section{Citrate@GNP}
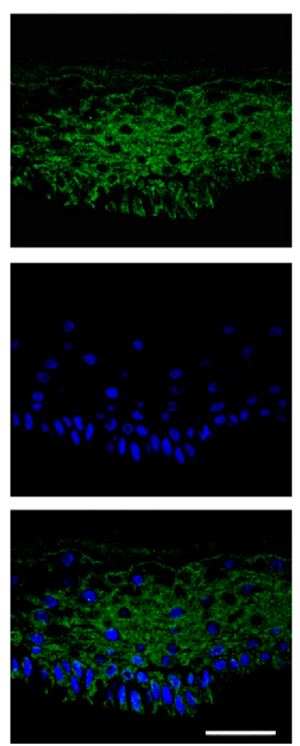

Figure 5. Influence of GNP exposure on the expression of tight junction proteins. (A) RT-PCR analysis for the transcriptional levels of tight junction proteins. (B) Western blot analysis of occludin expression in the epidermis $(* p<0.05$ ). (C) Immunofluorescence assay for occludin expression (occludin was labeled with green fluorescent probe, and nucleus were labeled with DAPI). Red frame indicates the intercellular space caused by CTAB@GNP exposure. Scale bars represent $50 \mu \mathrm{m}$. The samples were collected on exposure day 3.

compromised cell junctions would possibly offer direct penetration route for NPs. As the main components of the tight junction, ${ }^{50}$ the transcriptional levels of three biomarkers, including ZO-1, occludin, and claudin-1, were evaluated in different exposure groups. The results in Figure 5A illustrate that CTAB@GNP exposure causes significant elevation in the transcriptional level of occludin, but does not influence the other two (i.e., ZO-1 and claudin-1). As indicated, the claudins were corresponding to the pore way through which ions at the sizes of around $0.4 \mathrm{~nm}$ could diffuse, while occludin consisted of the leak way, regulating the macromolecular penetration. ${ }^{51}$ ZO-1 indirectly mediated tight junction by anchoring claudins and occludin to the cytoskeleton with PDZ and GUK domains. ${ }^{50}$ The increase in occludin mRNA suggested that CTAB@GNP influenced the leak way, which could contribute to the penetration of the NPs. In contrast to CTAB@GNP, neither PVP@GNP nor Citrate@GNP changed the transcriptional levels of the tested biomarkers (Figure 5A), revealing that they could have little effect on the tight junction of keratinocytes. To further confirm GNP induced effects on the expression of occludin, the exposed epidermis samples were submitted to Western blot analysis, and the results confirmed that CTAB@GNP notably increased the occludin protein level, while the other two GNPs had no effects (Figure 5B), which was well consistent with the results in Figure 5A.

The immunofluorescence experiment was further performed to characterize occludin location in keratinocytes. As indicated in Figure 5C, occludin stained with green fluorescence was mainly lined in the cytomembrane of the control, forming the tight layered keratinocytes with clear nuclei stained with DAPI locating in the center. In contrast to the hollow structure probed by occludin in the control, the widespread of this protein was observed in the cytoplasm of CTAB@GNP exposed keratinocytes, showing cellular internalization of occludin occurred in this group. ${ }^{52}$ Interspaces marked by red frame appeared in the adjacent cytomembranes. Nevertheless, this effect was not obvious in the other two exposure groups, and their occludin distribution was mainly located in cytomembrane, which was similar to the control. The intercellular space revealed by immunostaining of occludin absence in cell junction area in CTAB@GNP exposure group was not necessary detectable in normal histopathological observation due to its noncytotoxic stimulation and the intact tissue structure. According to the previously established 
(A)

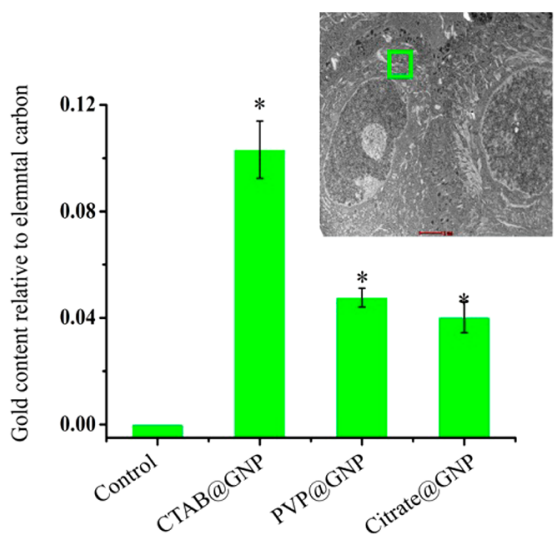

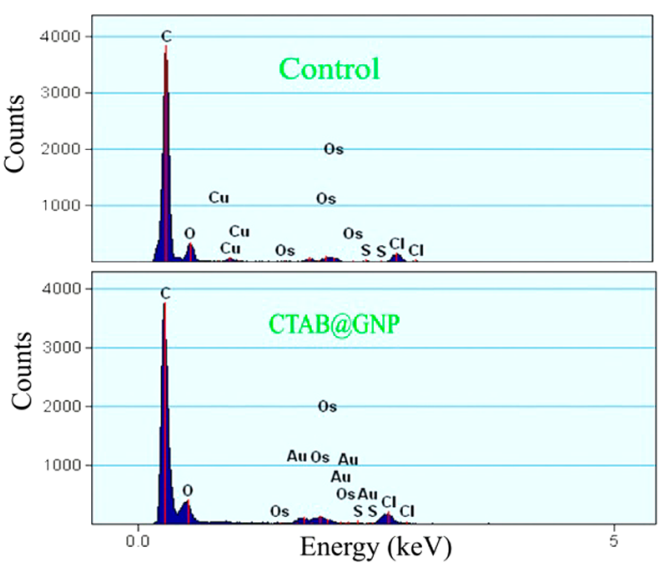

(C)
(B)
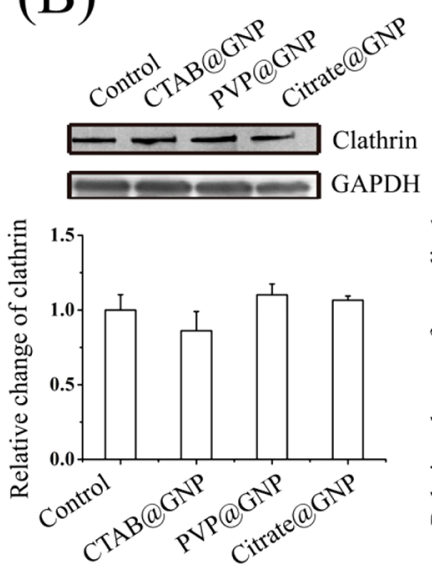
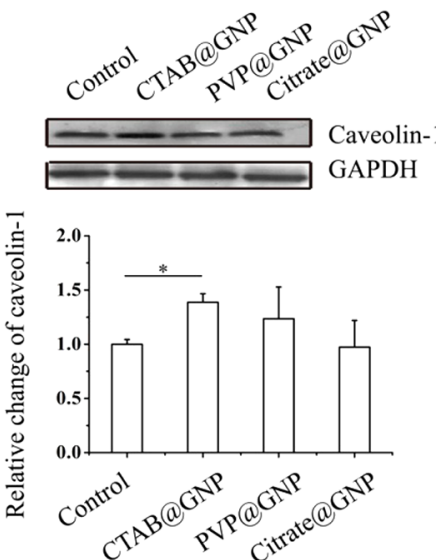

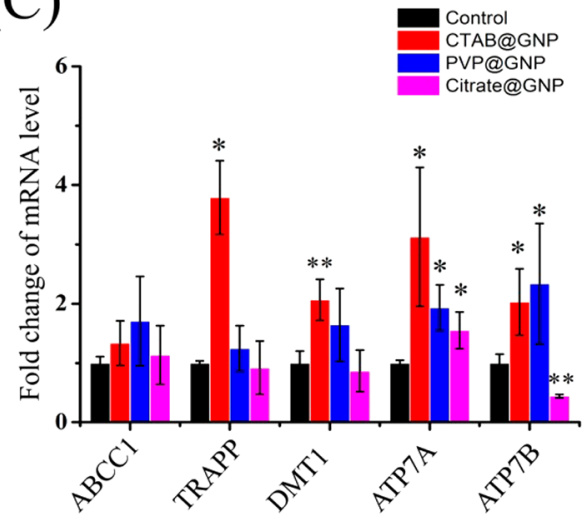

Figure 6. GNP penetration through tanscytosis pathway. (A) STEM-EDS characterization of Au distribution in keratinocytes. The green frame in the STEM graph indicates cytoplasmic space. The underlying histogram shows the comparison of gold contents adjusted by element carbon in different groups. The right panel is the representative EDS spectra of the control and CTAB@GNP exposure groups, showing the occurrence of Au signals in the epidermic samples due to GNP treatments. (B) Western blot assay for clathrin and caveolin-1 expressions in keratinocytes. (C) The relative transcriptional levels of the target exocytosis-related proteins. $* p<0.05$, $* *<0.001$. The samples were collected on exposure day 3 .

knowledge, $^{53}$ occludin in tight junction contained two extracellular helix domains, and they were connected with those of adjacent occludin molecular. The C-terminal of occludin, as the intracellular domain, was linked with ZO-1 in a phosphorylation-dependent pathway, through which occludin was anchored in cell membrane, sealing the paracellular gap of keratinocytes. Loss of the binding of occludin with ZO-1 would cause its dispersion in cytoplasm and open of paracellular space. CTAB@GNP induced the disintegration of occludin in cytomembrane, and the subsequent internalization would explain their penetration pathway through the Episkin model. The high regulation of occludin at both transcriptional and protein levels could be contributed by the intricate cellular regulatory network. The stable distribution of membrane occludin in PVP@GNP and Citrate@GNP exposure groups suggested normal function of the tight junction in keratinocytes, ${ }^{54,55}$ indicating little paracellular route appeared for NPs penetration. This finding was consistent with the trend of protein upward osmosis observed in Figure 3, showing CTAB@GNP induced the strongest effect on Episkin permeability among the three tested GNPs.

3.6. GNP Penetration through Transcytosis Pathway. Besides the paracellular route, NPs could possibly realize the penetration of the Episkin model through transcytosis pathway. To confirm if cellular internalization of GNPs occurred, STEMEDS was used to determine Au distribution in keratinocytes. As indicated in Figure 6A, the cytoplasm was qualitatively analyzed for elemental gold contents, and the results were adjusted by elemental carbon contents. It was obvious that GNP exposure significantly induced elevation in cytoplasmic gold contents when compared to the control $(p<0.05)$, showing the existence of endocytosis process. Gold levels in CTAB@GNP exposure groups were comparatively higher than those in PVP@GNP and Citrate@GNP exposure groups. This trend was consistent with the results in Figure $2 \mathrm{~A}$. Nevertheless, the difference of gold contents between CTAB@GNP and the other two exposure groups in Figure 6A was not as big as that in Figure 2A, which suggested the paracellular penetration of CTAB@GNP observed in Figure 5 also greatly contributed to its permeation through the Episkin. Although GNP images at the single-particle resolution would give more solid evidence on the penetration behavior of the tested nanoparticles, the rigidity of the specimen failed to tolerate the electron beam of STEM with higher energy under large magnification, suggesting the challenges in further studies on the improvement of the related analytical technique.

As for the process of endocytosis, three manners, including clathrin-mediated endocytosis, caveolae-mediated internalization, and clathrin or caveolea-independent internalization, were mainly involved. ${ }^{56}$ The endocytosis based on the use of chemical inhibitors and low temperature control could trigger some other cellular responses, besides the blockage of 
endocytosis, ${ }^{57}$ thus complicating the biological processes. The expressions of clathrin and caveolin have been extensively studied, ${ }^{58-61}$ and the high potentials of these protein biomarkers have been implicated in the explanation of cellular endocytosis. Herein, the investigation on both clathrin and caveolin-1 expression showed that no significant changes occurred in most exposure groups ( $p>0.05$, Figure 6B), except a little increase of caveolin-1 in the CTAB@GNP exposure group. Apparently, only caveolin-1 might play a slight role in endocytosis of CTAB@GNP, and the potential cellular internalization of GNPs as indicated by increased $\mathrm{Au}$ levels in Episkin tissues (Figures 2A and 6A) could be mainly regulated by a clathrin or caveolea-independent pathway. As indicated previously, ${ }^{62}$ the surface curvature of cells mediated clathrin or caveolae-dependent cellular internalization of NPs, via which the allowable penetration particle sizes were around $5 \mathrm{~nm}$ or more. Those smaller than $5 \mathrm{~nm}$ may be transported into cells through other pathways. ${ }^{63}$ Although the particle sizes of three GNPs tested herein were qualified ( $\geq 5 \mathrm{~nm}$, Figure 1$)$, the critical sizes of both Citrate@GNP and PVP@GNP (5.10 and $5.85 \mathrm{~nm}$ ) could induce less effects on clathrin or caveolaedependent cellular internalization than did CTAB@GNP (8.76 $\mathrm{nm})$. In addition, their surface functionalization with different charges could greatly influence their contact with cellular biomembrane. ${ }^{64}$ As compared to negatively or neutrally charged NPs, positively charged NPs could break through the cytomembrane more easily, ${ }^{65-67}$ and some negatively charged NPs could be transferred into cells through the interaction with some positive areas in membranes. 57

The exocytosis of GNPs could also be an important process, influencing their penetration behaviors through the Episkin. In general, ABCC1, TRAPP, ATP7A, ATP7B, and DMT1 are responsible for transporting and exporting metal ions or particles. $^{68,69}$ The evaluation of the transcriptional levels of these five biomarkers showed CTAB@GNP significantly increased TRAPP, DMT1, ATP7A, and STP7B mRNA expressions $(p<0.05$ or 0.01 , Figure $6 \mathrm{C})$, but did not affect ABCC1 expression. In contrast to CTAB@GNP, the PVP@ GNP only significantly increased the transcriptional levels of ATP7A and ATP7B $(p<0.05)$, while Citrate@GNP slightly increased ATP7A mRNA level $(p<0.05)$, but significantly decreased ATP7A mRNA level $(p<0.01$, Figure 6C). This result suggested that positively charged GNPs could be exported from cells with the help of vesicle secretion-related protein and metal transporters, and those with neutrally or negatively charged surfaces could experience exocytosis process with metal transporters involved. The distinctive responses due to the treatments of GNPs with unique characteristics indicated intricate cellular exocytosis processes were induced in keratinocytes.

\section{CONCLUSION}

In summary, this research revealed the distinctive penetration behaviors of three GNPs based on human reconstructed 3D Episkin model due to their different surface functionalization. Although all GNPs could induce the phase change of lipid lamella and pass through the epidermis, positively charged GNPs exhibited the most efficient skin penetration through both the paracellular routes and the transcellular pathway, when compared to neutrally or negatively charged GNPs. The surface functionalization-regulated GNP penetration in Episkin has provided substantial evidence on the biosafety or bioefficiency evaluation for NPs with different coatings in cosmetics and some other transdermal pharmaceutical development, and the $3 \mathrm{D}$ epidermis platform has also demonstrated very promising in skin-related studies on emerging nanomaterial penetration. The information on the biological effects regulated by a systematic variation of the physiochemical factors, like surface charge or particle size, would be highly encouraged for the perspective of nanotechnology improvement in future studies.

\section{ASSOCIATED CONTENT}

\section{Supporting Information}

The Supporting Information is available free of charge on the ACS Publications website at DOI: 10.1021/acsami.7b13700.

Figure S1, schematic diagram of human epidermis; Figure S2, outline of 3D Episkin model; Figure S3, laser ablation ICP-MS results; Figure S4, histopathological section graphs; Table S1, quality control of the Episkin model obtained from the vendor; Table S2, instrumental parameters for laser ablation ICP-MS analysis; Table S3, parameters for protein identification; Table S4, primer sequences; Table S5, zeta potentials and hydrated particle sizes of three GNPs; and Table S6, zeta potentials and hydrated particle sizes of three GNPs after 7-day storage (PDF)

\section{AUTHOR INFORMATION}

\section{Corresponding Author}

*Tel.: +86-10-62849334. Fax: +86-10-62849339. E-mail: zhouqf@rcees.ac.cn.

ORCID $\odot$

Qian S. Liu: 0000-0003-4759-5016

Qunfang Zhou: 0000-0003-2521-100X

Notes

The authors declare no competing financial interest.

\section{ACKNOWLEDGMENTS}

This study was financially supported by the National Natural Science Foundation of China (21477153, 21461142001, and 21621064), and the Chinese Academy of Science (14040302, QYZDJ-SSW-DQC017).

\section{REFERENCES}

(1) Heuer-Jungemann, A.; Harimech, P. K.; Brown, T.; Kanaras, A. G. Gold nanoparticles and fluorescently-labelled DNA as a platform for biological sensing. Nanoscale 2013, 5 (20), 9503-9510.

(2) Webb, J. A.; Bardhan, R. Emerging advances in nanomedicine with engineered gold nanostructures. Nanoscale 2014, 6 (5), 25022530.

(3) Papakostas, D.; Rancan, F.; Sterry, W.; Blume-Peytavi, U.; Vogt, A. Nanoparticles in dermatology. Arch. Dermatol. Res. 2011, 303 (8), $533-550$.

(4) Shi, J.; Kantoff, P. W.; Wooster, R.; Farokhzad, O. C. Cancer nanomedicine: progress, challenges and opportunities. Nat. Rev. Cancer 2016, 17, 20.

(5) Morishita, Y.; Yoshioka, Y.; Takimura, Y.; Shimizu, Y.; Namba, Y.; Nojiri, N.; Ishizaka, T.; Takao, K.; Yamashita, F.; Takuma, K.; Ago, Y.; Nagano, K.; Mukai, Y.; Kamada, H.; Tsunoda, S.-i.; Saito, S.; Matsuda, T.; Hashida, M.; Miyakawa, T.; Higashisaka, K.; Tsutsumi, Y. Distribution of Silver Nanoparticles to Breast Milk and Their Biological Effects on Breast-Fed Offspring Mice. ACS Nano 2016, $10,8180$.

(6) Wen, R.; Yang, X.; Hu, L.; Sun, C.; Zhou, Q.; Jiang, G. Braintargeted distribution and high retention of silver by chronic intranasal 
instillation of silver nanoparticles and ions in Sprague-Dawley rats. J. Appl. Toxicol. 2016, 36 (3), 445-453.

(7) Lankveld, D.; Oomen, A.; Krystek, P.; Neigh, A.; Troost-de Jong, A.; Noorlander, C.; Van Eijkeren, J.; Geertsma, R.; De Jong, W. The kinetics of the tissue distribution of silver nanoparticles of different sizes. Biomaterials 2010, 31 (32), 8350-8361.

(8) De Jong, W. H.; Hagens, W. I.; Krystek, P.; Burger, M. C.; Sips, A. J.; Geertsma, R. E. Particle size-dependent organ distribution of gold nanoparticles after intravenous administration. Biomaterials 2008, 29 (12), 1912-1919.

(9) Tang, J.; Xiong, L.; Wang, S.; Wang, J.; Liu, L.; Li, J.; Yuan, F.; Xi, T. Distribution, translocation and accumulation of silver nanoparticles in rats. J. Nanosci. Nanotechnol. 2009, 9 (8), 4924-4932.

(10) Fabian, E.; Landsiedel, R.; Ma-Hock, L.; Wiench, K.; Wohlleben, W.; Van Ravenzwaay, B. Tissue distribution and toxicity of intravenously administered titanium dioxide nanoparticles in rats. Arch. Toxicol. 2008, 82 (3), 151-157.

(11) Rancan, F.; Gao, Q.; Graf, C.; Troppens, S.; Hadam, S.; Hackbarth, S.; Kembuan, C.; Blume-Peytavi, U.; Rühl, E.; Lademann, J. r. Skin penetration and cellular uptake of amorphous silica nanoparticles with variable size, surface functionalization, and colloidal stability. ACS Nano 2012, 6 (8), 6829-6842.

(12) Labouta, H. I.; Schneider, M. Interaction of inorganic nanoparticles with the skin barrier: current status and critical review. Nanomedicine 2013, 9 (1), 39-54.

(13) Yang, Y.; Sunoqrot, S.; Stowell, C.; Ji, J.; Lee, C.-W.; Kim, J. W.; Khan, S. A.; Hong, S. Effect of size, surface charge, and hydrophobicity of poly (amidoamine) dendrimers on their skin penetration. Biomacromolecules 2012, 13 (7), 2154-2162.

(14) Holmes, A. M.; Song, Z.; Moghimi, H. R.; Roberts, M. S. Relative Penetration of Zinc Oxide and Zinc Ions into Human Skin after Application of Different Zinc Oxide Formulations. ACS Nano 2016, 10, 1810.

(15) Fernandes, R.; Smyth, N. R.; Muskens, O. L.; Nitti, S.; HeuerJungemann, A.; Ardern-Jones, M. R.; Kanaras, A. G. Interactions of Skin with Gold Nanoparticles of Different Surface Charge, Shape, and Functionality. Small 2015, 11 (6), 713-721.

(16) Mortensen, L.; Zheng, H.; Faulknor, R; De Benedetto, A.; Beck, L.; DeLouise, L. A. Increased in Vivo Skin Penetration of Quantum Dots with UVR and in Vitro Quantum Dot Cytotoxicity; Proc. SPIE, 2009; p 718919.

(17) Mortensen, L. J.; Oberdörster, G.; Pentland, A. P.; DeLouise, L. A. In Vivo Skin Penetration of Quantum Dot Nanoparticles in the Murine Model: The Effect of UVR. Nano Lett. 2008, 8 (9), 27792787.

(18) Mavon, A.; Miquel, C.; Lejeune, O.; Payre, B.; Moretto, P. In vitro percutaneous absorption and in vivo stratum corneum distribution of an organic and a mineral sunscreen. Skin pharmacology and physiology 2006, 20 (1), 10-20.

(19) Wu, J.; Liu, W.; Xue, C.; Zhou, S.; Lan, F.; Bi, L.; Xu, H.; Yang, X.; Zeng, F.-D. Toxicity and penetration of $\mathrm{TiO} 2$ nanoparticles in hairless mice and porcine skin after subchronic dermal exposure. Toxicol. Lett. 2009, 191 (1), 1-8.

(20) Huang, Y.; Yu, F.; Park, Y.-S.; Wang, J.; Shin, M.-C.; Chung, H. S.; Yang, V. C. Co-administration of protein drugs with gold nanoparticles to enable percutaneous delivery. Biomaterials 2010, 31 (34), 9086-9091.

(21) Chu, M.; Wu, Q.; Wang, J.; Hou, S.; Miao, Y.; Peng, J.; Sun, Y. In vitro and in vivo transdermal delivery capacity of quantum dots through mouse skin. Nanotechnology 2007, 18 (45), 455103.

(22) Labouta, H. I.; El-Khordagui, L. K.; Kraus, T.; Schneider, M. Mechanism and determinants of nanoparticle penetration through human skin. Nanoscale 2011, 3 (12), 4989-4999.

(23) Gupta, R.; Rai, B. Penetration of Gold Nanoparticles through Human Skin: Unraveling Its Mechanisms at the Molecular Scale. J. Phys. Chem. B 2016, 120 (29), 7133-7142.

(24) Netzlaff, F.; Kaca, M.; Bock, U.; Haltner-Ukomadu, E.; Meiers, P.; Lehr, C.-M.; Schaefer, U. F. Permeability of the reconstructed human epidermis model Episkin ${ }^{\circledR}$ in comparison to various human skin preparations. Eur. J. Pharm. Biopharm. 2007, 66 (1), 127-134.

(25) Engesland, A.; Skalko-Basnet, N.; Flaten, G. E. Phospholipid Vesicle-Based Permeation Assay and EpiSkin ${ }^{\circledR}$ in Assessment of Drug Therapies Destined for Skin Administration. J. Pharm. Sci. 2015, 104 (3), 1119-1127.

(26) Abdallah, M. A.-E.; Pawar, G.; Harrad, S. Evaluation of 3Dhuman skin equivalents for assessment of human dermal absorption of some brominated flame retardants. Environ. Int. 2015, 84, 64-70.

(27) Alkilany, A. M.; Murphy, C. J. Gold Nanoparticles with a Polymerizable Surfactant Bilayer: Synthesis, Polymerization, and Stability Evaluation. Langmuir 2009, 25 (24), 13874-13879.

(28) Cozad, M. J.; Bachman, S. L.; Grant, S. A. Assessment of decellularized porcine diaphragm conjugated with gold nanomaterials as a tissue scaffold for wound healing. J. Biomed. Mater. Res., Part A 2011, 99A (3), 426-434.

(29) Wang, S.; Lu, W.; Tovmachenko, O.; Rai, U. S.; Yu, H.; Ray, P. C. Challenge in understanding size and shape dependent toxicity of gold nanomaterials in human skin keratinocytes. Chem. Phys. Lett. 2008, 463 (1), 145-149.

(30) Lee, O.; Jeong, S. H.; Shin, W. U.; Lee, G.; Oh, C.; Son, S. W. Influence of surface charge of gold nanorods on skin penetration. Skin Research and Technology 2013, 19 (1), e390-e396.

(31) Zhao, X.; Lu, D.; Liu, S. Q.; Li, Y.; Feng, R.; Hao, F.; Qu, G.; Zhou, Q.; Jiang, G. Hematological Effects of Gold Nanorods on Erythrocytes: Hemolysis and Hemoglobin Conformational and Functional Changes. Advanced Science 2017, 1700296-n/a.

(32) Calzolai, L.; Franchini, F.; Gilliland, D.; Rossi, F. ProteinNanoparticle Interaction: Identification of the Ubiquitin-Gold Nanoparticle Interaction Site. Nano Lett. 2010, 10 (8), 3101-3105.

(33) Ali, S. M.; Yosipovitch, G. Skin pH: from basic science to basic skin care. Acta dermato-venereologica 2013, 93 (3), 261-269.

(34) Wertz, P. W. Lipids and barrier function of the skin. Acta Dermato-Venereologica 2000, 80, 7.

(35) Freese, C.; Uboldi, C.; Gibson, M. I.; Unger, R. E.; Weksler, B. B.; Romero, I. A.; Couraud, P.-O.; Kirkpatrick, C. J. Uptake and cytotoxicity of citrate-coated gold nanospheres: comparative studies on human endothelial and epithelial cells. Part. Fibre Toxicol. 2012, 9 (1), 23.

(36) Gangwar, R. K.; Dhumale, V. A.; Kumari, D.; Nakate, U. T.; Gosavi, S. W.; Sharma, R. B.; Kale, S. N.; Datar, S. Conjugation of curcumin with PVP capped gold nanoparticles for improving bioavailability. Mater. Sci. Eng., C 2012, 32 (8), 2659-2663.

(37) Schneider, M.; Stracke, F.; Hansen, S.; Schaefer, U. F. Nanoparticles and their interactions with the dermal barrier. Derm.Endocrinol. 2009, 1 (4), 197-206.

(38) Gennari, C. G. M.; Franzè, S.; Pellegrino, S.; Corsini, E.; Vistoli, G.; Montanari, L.; Minghetti, P.; Cilurzo, F. Skin Penetrating Peptide as a Tool to Enhance the Permeation of Heparin through Human Epidermis. Biomacromolecules 2016, 17 (1), 46-55.

(39) Cilurzo, F.; Vistoli, G.; Selmin, F.; Gennari, C. G. M.; Musazzi, U. M.; Franzé, S.; Lo Monte, M.; Minghetti, P. An Insight into the Skin Penetration Enhancement Mechanism of N-Methylpyrrolidone. Mol. Pharmaceutics 2014, 11 (3), 1014-1021.

(40) Mendelsohn, R.; Flach, C. R.; Moore, D. J. Determination of molecular conformation and permeation in skin via IR spectroscopy, microscopy, and imaging. Biochim. Biophys. Acta, Biomembr. 2006, 1758 (7), 923-933.

(41) Karande, P.; Jain, A.; Ergun, K.; Kispersky, V.; Mitragotri, S. Design principles of chemical penetration enhancers for transdermal drug delivery. Proc. Natl. Acad. Sci. U. S. A. 2005, 102 (13), 46884693.

(42) Wang, B.; Zhang, L.; Bae, S. C.; Granick, S. Nanoparticleinduced surface reconstruction of phospholipid membranes. Proc. Natl. Acad. Sci. U. S. A. 2008, 105 (47), 18171-18175.

(43) Somerharju, P.; Virtanen, J. A.; Cheng, K. H. Lateral organisation of membrane lipids: the superlattice view. Biochim. Biophys. Acta, Mol. Cell Biol. Lipids 1999, 1440 (1), 32-48. 
(44) Silva, C.; Nunes, S.; Eusebio, M.; Sousa, J.; Pais, A. Study of human stratum corneum and extracted lipids by thermomicroscopy and DSC. Chem. Phys. Lipids 2006, 140 (1), 36-47.

(45) Al-Saidan, S.; Barry, B.; Williams, A. Differential scanning calorimetry of human and animal stratum corneum membranes. Int. J. Pharm. 1998, 168 (1), 17-22.

(46) Bouwstra, J.; Gooris, G.; Weerheim, A.; Kempenaar, J.; Ponec, M. Characterization of stratum corneum structure in reconstructed epidermis by X-ray diffraction. J. Lipid Res. 1995, 36, 496-504.

(47) Pino, C. J.; Gutterman, J. U.; Vonwil, D.; Mitragotri, S.; Shastri, V. P. Glycosylation facilitates transdermal transport of macromolecules. Proc. Natl. Acad. Sci. U. S. A. 2012, 109 (52), 21283-21288.

(48) Kubo, A.; Nagao, K.; Yokouchi, M.; Sasaki, H.; Amagai, M. External antigen uptake by Langerhans cells with reorganization of epidermal tight junction barriers. J. Exp. Med. 2009, 206 (13), 29372946.

(49) Schneeberger, E. E.; Lynch, R. D. The tight junction: a multifunctional complex. American Journal of Physiology - Cell Physiology 2004, 286 (6), C1213-C1228.

(50) Zihni, C.; Mills, C.; Matter, K.; Balda, M. S. Tight junctions: from simple barriers to multifunctional molecular gates. Nat. Rev. Mol. Cell Biol. 2016, 17, 564.

(51) Shen, L.; Weber, C. R.; Raleigh, D. R.; Yu, D.; Turner, J. R. Tight junction pore and leak pathways: a dynamic duo. Annu. Rev. Physiol. 2011, 73, 283-309.

(52) Stamatovic, S. M.; Keep, R. F.; Wang, M. M.; Jankovic, I.; Andjelkovic, A. V. Caveolae-mediated internalization of occludin and claudin-5 during CCL2-induced tight junction remodeling in brain endothelial cells. J. Biol. Chem. 2009, 284 (28), 19053-19066.

(53) Tash, B. R.; Bewley, M. C.; Russo, M.; Keil, J. M.; Griffin, K. A.; Sundstrom, J. M.; Antonetti, D. A.; Tian, F.; Flanagan, J. M. The occludin and ZO-1 complex, defined by small angle X-ray scattering and NMR, has implications for modulating tight junction permeability. Proc. Natl. Acad. Sci. U. S. A. 2012, 109 (27), 10855-10860.

(54) Phillips, B. E.; Cancel, L.; Tarbell, J. M.; Antonetti, D. A. Occludin independently regulates permeability under hydrostatic pressure and cell division in retinal pigment epithelial cells. Invest. Ophthalmol. Visual Sci. 2008, 49 (6), 2568-2576.

(55) Furuse, M.; Hata, M.; Furuse, K.; Yoshida, Y.; Haratake, A.; Sugitani, Y.; Noda, T.; Kubo, A.; Tsukita, S. Claudin-based tight junctions are crucial for the mammalian epidermal barrier a lesson from claudin-1-deficient mice. J. Cell Biol. 2002, 156 (6), 1099-1111.

(56) McMahon, H. T.; Boucrot, E. Molecular mechanism and physiological functions of clathrin-mediated endocytosis. Nat. Rev. Mol. Cell Biol. 2011, 12 (8), 517-533.

(57) Verma, A.; Stellacci, F. Effect of Surface Properties on Nanoparticle-Cell Interactions. Small 2010, 6 (1), 12-21.

(58) Voigt, J.; Christensen, J.; Shastri, V. P. Differential uptake of nanoparticles by endothelial cells through polyelectrolytes with affinity for caveolae. Proc. Natl. Acad. Sci. U. S. A. 2014, 111 (8), 2942-2947.

(59) Ekkapongpisit, M.; Giovia, A.; Follo, C.; Caputo, G.; Isidoro, C. Biocompatibility, endocytosis, and intracellular trafficking of mesoporous silica and polystyrene nanoparticles in ovarian cancer cells: effects of size and surface charge groups. Int. J. Nanomed. 2012, 7, 4147-4158.

(60) Hu, Z.; Pan, Y.; Wang, J.; Chen, J.; Li, J.; Ren, L. Meso-tetra (carboxyphenyl) porphyrin (TCPP) nanoparticles were internalized by SW480 cells by a clathrin-mediated endocytosis pathway to induce high photocytotoxicity. Biomed. Pharmacother. 2009, 63 (2), 155-164.

(61) Coradeghini, R.; Gioria, S.; García, C. P.; Nativo, P.; Franchini, F.; Gilliland, D.; Ponti, J.; Rossi, F. Size-dependent toxicity and cell interaction mechanisms of gold nanoparticles on mouse fibroblasts. Toxicol. Lett. 2013, 217 (3), 205-216.

(62) Nel, A. E.; Madler, L.; Velegol, D.; Xia, T.; Hoek, E. M. V.; Somasundaran, P.; Klaessig, F.; Castranova, V.; Thompson, M. Understanding biophysicochemical interactions at the nano-bio interface. Nat. Mater. 2009, 8 (7), 543-557.

(63) Zhang, S.; Gao, H.; Bao, G. Physical principles of nanoparticle cellular endocytosis. ACS Nano 2015, 9 (9), 8655-8671.
(64) Verma, A.; Uzun, O.; Hu, Y.; Hu, Y.; Han, H.-S.; Watson, N.; Chen, S.; Irvine, D. J.; Stellacci, F. Surface-structure-regulated cellmembrane penetration by monolayer-protected nanoparticles. Nat. Mater. 2008, 7 (7), 588-595.

(65) Chen, L.; Mccrate, J. M.; Lee, J. C.; Li, H. The role of surface charge on the uptake and biocompatibility of hydroxyapatite nanoparticles with osteoblast cells. Nanotechnology 2011, 22 (10), 105708.

(66) Chung, T.-H.; Wu, S.-H.; Yao, M.; Lu, C.-W.; Lin, Y.-S.; Hung, Y.; Mou, C.-Y.; Chen, Y.-C.; Huang, D.-M. The effect of surface charge on the uptake and biological function of mesoporous silica nanoparticles in 3T3-L1 cells and human mesenchymal stem cells. Biomaterials 2007, 28 (19), 2959-2966.

(67) He, C.; Hu, Y.; Yin, L.; Tang, C.; Yin, C. Effects of particle size and surface charge on cellular uptake and biodistribution of polymeric nanoparticles. Biomaterials 2010, 31 (13), 3657-3666.

(68) Wang, L.; Zhang, T.; Li, P.; Huang, W.; Tang, J.; Wang, P.; Liu, J.; Yuan, Q.; Bai, R.; Li, B.; Zhang, K.; Zhao, Y.; Chen, C. Use of Synchrotron Radiation-Analytical Techniques To Reveal Chemical Origin of Silver-Nanoparticle Cytotoxicity. ACS Nano 2015, 9 (6), 6532-6547.

(69) Pillai, S.; Behra, R.; Nestler, H.; Suter, M. J.-F.; Sigg, L.; Schirmer, K. Linking toxicity and adaptive responses across the transcriptome, proteome, and phenotype of Chlamydomonas reinhardtii exposed to silver. Proc. Natl. Acad. Sci. U. S. A. 2014, 111 (9), 3490-3495. 